\title{
Study on 3D Reconstruction of Defocus Blur Light Spots based on Al-enabled Virtual - Wireless Communications
}

\author{
Yun Shi \\ Nanjing University of Aeronautics and Astronautics \\ Yan-yan Zhu (D710667612@qq.com ) \\ Nanjing University of Aeronautics and Astronautics
}

\section{Research}

Keywords: Defocus blur radius, light spot, double hidden layer BP network model, 3D reconstruction

Posted Date: April 28th, 2021

DOl: https://doi.org/10.21203/rs.3.rs-458082/v1

License: (c) (1) This work is licensed under a Creative Commons Attribution 4.0 International License.

Read Full License 


\title{
Study on 3D Reconstruction of Defocus Blur Light Spots bas ed on AI-enabled Virtual - Wireless Communications
}

\author{
Shi Yun ${ }^{1,2}$, Zhu Yan-yan ${ }^{2 *}$ \\ 1. College of Mechanical \& Electrical Engineering, Nanjing University of Aeronautics \& Astronautics, \\ Nanjing, 210016 China. \\ 2. School of Electronics and Information Engineering, West Anhui University, Lu' an, 237012, China. \\ Corresponding Author' s: Zhu Yan-yan, Email: 710667612@qq.com
}

\begin{abstract}
:
The application of artificial intelligence and machine learning technology in the field of wireless communication has received great attention. The success of artificial intelligence in speech understanding, image recognition, natural language processing and other fields shows its great potential to solve the problem of difficult modeling. Aiming at the problem of $3 \mathrm{D}$ reconstruction of defocused blur spots, a method based on adaptive defocus blur radius estimation and double hidden layer BP neural network is proposed. This method first uses an adaptive segmentation algorithm to extract the approximate elliptical spot in the defocused blurred image, and then uses the gradient amplitude distribution to extract the defocused blurred area, thereby calculating the blur radius. Then, using the network to adaptively learn the geometric structure relationship between the target $3 \mathrm{D}$ position, the target image point position and the defocus blur radius, it is established that the center pixel coordinates of the light spot and the corresponding defocus blur radius are used as input, and the $3 \mathrm{D}$ coordinates of the target are used as input. Output, the two hidden layers in the middle have established a neural network for the $3 \mathrm{D}$ reconstruction of the defocused blur spot. The experimental results show that the double hidden layer BP network model proposed in this paper can realize the $3 \mathrm{D}$ reconstruction of the blurred spot after training, and the method proposed in this paper has higher 3D reconstruction accuracy than without considering the defocus blur effect.
\end{abstract}

Keywords: Defocus blur radius, light spot, double hidden layer BP network model, 3D reconstruction

\section{Introduction}

In the actual shooting process, due to the limited depth of field of the imaging system, the collected images will have varying degrees of defocus blur. Defocus blur leads to image degradation and loss of image detail and texture information, which will seriously affect the visual perception of the image and the accuracy of 3D reconstruction. Therefore, accurately estimating the defocus model parameters is of great significance to restore image details, improve image quality and 3D reconstruction accuracy.

This paper studies the 3D reconstruction of light spots under defocusing blur. First, the principle of defocus blur formation is introduced. Based on the disc defocus blur model, after threshold processing, the defocus blur spot is extracted by the gradient amplitude post-filling process, and the defocus blur radius is obtained. Then, use the double hidden layer BP neural network to take the center pixel coordinates of the light spot in the defocused blurred image and the corresponding defocus radius as the input layer of the network, and the 3D coordinates of the light spot as the output layer for training, to obtain the $3 \mathrm{D}$ reconstruction network of the defocused blurred targe. In this method, as long as the center position of a spot in the multi-eye image and the blur radius in each image are given, the corresponding three-dimensional coordinates can be obtained according to the trained network model. The reconstruction result considering the defocus radius is compared with the result of direct reconstruction without considering the defocus radius. The results show that the $3 \mathrm{D}$ reconstruction accuracy of the algorithm proposed in this paper is higher than that of the $3 \mathrm{D}$ reconstruction result without considering the defocus blur radius.

\section{Method}

\section{1Defocus blur radius estimation}

The loss of defocused image details is serious, and the image texture is blurred, which is not conducive to the development of follow-up research and related vision applications. At present, scholars at home and abroad have carried out a lot of research on the parameter estimation of the defocus model, and many fuzzy estimation algorithms have been continuously proposed. Literature [1] uses Wiener filtering, combined with the frequency domain zero point method to estimate the defocus blur radius. This method performs well for images with high signal-to-noise ratio. When the noise is too large, the first dark spot is easily detected to fail. It is difficult to calculate the degradation parameters. Literature [2] first calculates the amplitude response of the image, and estimates the blur radius by detecting the dark spot in the amplitude response, but the algorithm needs to set a proper threshold to remove noise 
and obtain dark spots, so its universality is poor. Literature [3] proposed to use the Hopfield neural network sub-optimal algorithm to reconstruct the image when solving the blind restoration of remote sensing images, while the fuzzy radius estimation adopts the differential autocorrelation method. The algorithm obtains a higher image definition, but for low signal noise Compared with the situation, its adaptability is poorer. Literature [4] uses the image edge fuzzy spectrum feature to estimate the blur radius. When the image edge feature is not obvious, the algorithm is poor. Literature [5] uses RBF neural network combined with wavelet domain features to estimate fuzzy parameters, its algorithm is complicated and the calculation efficiency is low. Literature [6] proposed a semi-blind deconvolution method for defocused images based on RBF neural network and iterative Wiener filtering, and trained the RBF neural network in the wavelet domain to estimate the defocus parameters. Literature [7] proposed a robust noise independent model to estimate fuzzy parameters, which requires multiple iterations to calculate the Bessel function, so the algorithm complexity is high.

Literature [8] combines the gradient image and its second derivative to identify the blur radius by judging the sign change of the second derivative in the direction of the gradient edge. Since the edge cannot be guaranteed to be a step edge, the error is relatively large. Literature [9] uses wavelet transform and biological optimization algorithm to solve the optimal blur radius. In actual calculation, multiple iterations are required, so the complexity is high. Literature [10] uses the linear edges in the image and combines the statistical characteristics of step edge diffusion to derive the transformation model between the line spread function and the defocus blur parameters to estimate the defocus degradation model parameters. The algorithm is suitable for single and different depth of field scenes. However, its complexity is relatively high, and it is not practical to judge whether the edge spread function is S-shaped manually. In addition, literature [11] calculated the local entropy of the degraded image through texture analysis, combined with statistical rules to estimate the line spread function, and finally calculated the blur radius.

In the defocus blur image restoration process, the point spread function can be used to simulate the defocus blur process. In practical applications, the disc defocus model can be used to simulate the degree of blur diffusion of the defocused image. The size of the disc corresponds to the defocus blur radius. Therefore, to establish the disc defocus model, the blur radius must first be estimated.

\subsection{Defocus model}

Ignoring the influence of noise, the spatial degradation model of defocus blur can be expressed as:

$$
g(x, y)=f(x, y) \otimes \delta(x, y)
$$

where, $g(x, y)$ is the real image with out-of-focus blur, $f(x, y)$ is the sharp image, $\delta(x, y)$ is the point spread function, $g(x, y)$ is the defocus blurred image formed by $f(x, y)$ through the $\delta(x, y)$ defocus imaging system, and $\otimes$ represents the convolution operation.

Commonly used defocus blurs include Gaussian defocus model and disc defocus model [12]. Because geometric optics can derive the disc defocus model, and the model is simple, this paper uses the disc defocus model, and its specific model can be expressed by equation (2).

$$
\delta(x, y)=\left\{\begin{array}{cc}
1 /\left(\pi R^{2}\right) & \sqrt{x^{2}+y^{2}} \leq R \\
0 & \sqrt{x^{2}+y^{2}}>R
\end{array}\right.
$$

where, $R$ represents the defocus blur radius. The frequency domain expression is:

$$
H(u, v)=\frac{2 J_{1}\left(R \sqrt{\left(\frac{2 \pi u}{M}\right)^{2}+\left(\frac{2 \pi v}{N}\right)^{2}}\right)}{R \sqrt{\left(\frac{2 \pi u}{M}\right)^{2}+\left(\frac{2 \pi v}{N}\right)^{2}}}
$$

where, $J_{1}$ is the Bessel function of the first kind, and $M$ and $N$ are the image size. In fact, the trajectory of the zero-crossing point of the out-of-focus blurred image satisfies the circle equation [13], and the relationship between the spectral dark spot and the blur radius is as follows:

$$
R=\frac{z_{n} k}{2 \pi r_{R, n}}
$$

where, $r_{R, n}$ is the radius of the dark spot formed by the $n$ zero-crossing point, and its size is $k \times k$. $z_{n}$ is the nth zero of the Bessel function of the first kind. From equation (4), it can be seen that $R$ and $r_{R, n}$ are inversely proportional. 
Figure 1 shows the formation process of the defocus blur. Figure 1(a) shows the object point at a distance of $d_{f}$ (the focal length of the camera) from the lens. At this time, it will gather into a point on the imaging plane. If the object point is not at the focal plane of the camera, but the object point is placed in another position, the object point will be gathered on the imaging plane, not a point, regardless of whether the position of the object point is farther than the focal plane or closer than the focal plane of the lens $d$. It is the appearance of the Circle of Confusion ( $\mathrm{CoC})$. In Figure 1, $f_{0}$ is the focal length of the lens, $c$ is the diameter of the pixel $\mathrm{CoC}$, and its value indicates the degree of defocusing of the image. Figure 1(b) shows the $\mathrm{CoC}$ relationship diagram based on the disc defocus model. The diameter of the defocused disc in the figure is the diameter $c$ of the display pixel $\mathrm{CoC}$.

Figure 1 The relationship between the principle of defocus blur formation and $\mathrm{CoC}$

\subsection{Blur radius estimation}

This paper proposes a defocus blur radius estimation algorithm based on adaptive segmentation. The algorithm first uses the adaptive segmentation algorithm to extract and mark the circular area in the defocus blur image, and then uses the gradient operator to calculate the gradient amplitude, and pass the threshold Select and extract the defocus blur area, and then calculate the blur radius. The grayscale of the image in the defocus blurred area often shows a gradual change, so the gradient amplitude in this area has its particularity. For the non-circular confusion area, the gray value of the entire white scene is close to that of the gradient in this area. The amplitude is small. Based on this, an appropriate threshold can be set, and the fuzzy area can be obtained by extracting the gradient amplitude at the edge of the black and white junction of the light spot. It is not difficult to find that the obtained fuzzy area is similar to a circular ring. Suppose the area of the outer circle of the ring is $S_{1}$, the area of the inner circle is $S_{2}$, and the blur radius is, then:

$$
R=\sqrt{\frac{S_{1}}{\pi}}-\sqrt{\frac{S_{2}}{\pi}}
$$

In practice, multiple measurements can be taken to average to reduce system errors. Suppose the area sequence of the detected circle is $\left(\tilde{s}_{1}, s_{1}\right),\left(\tilde{s}_{2}, S_{2}\right), \cdots,\left(\tilde{s}_{n}, s_{n}\right)$, then the fuzzy radius of the system can be expressed as:

$$
R=\frac{1}{n} \sum_{i=1}^{n}\left(\sqrt{\frac{\tilde{s}_{i}}{\pi}}-\sqrt{\frac{s_{i}}{\pi}}\right)
$$

where $\tilde{s}_{1}, s_{1}$ represents the area of the outer circle and the inner circle respectively, and $n$ is the number of detected circles.

\subsection{Defocus blur radius estimation experiment}

In order to verify the effectiveness of the algorithm, four industrial cameras were used to simultaneously collect multiple sets of out-of-focus blurred images under different conditions. The shooting results are shown in Figure 2. It can be seen from Figure 2 that the size of the blurred spot set in the experiment is small, and its proportion in the whole image is small. In this paper, the defocus blur radius estimation algorithm based on adaptive segmentation has been used to obtain better results. The experiment is implemented by programming in the Windows 7 system Intel (R) Xeon (R) CP-W3565@3.20GHz, memory3.99GB environment. Figure 2 shows the 1067th actual imaging image in camera 1 obtained by synchronously shooting three light spots by four cameras, and the image size is all $1088 \times 1456$ pixels.

\section{Figure 2 Actual image taken}

Figure 2 The actual captured image contains the out-of-focus blurry spot with a green rectangular frame. After zooming in, see 3 on the right. Figure 2 The actual captured image contains the interference information with a red rectangular frame. After zooming in, see the upper right corner of Figure 2. 1, 2. First, the image is processed with interference information, and the threshold is set to 200. The image obtained after threshold processing is shown in Figure 3, and the interference information is completely eliminated.

Figure 3 Threshold processing result graph 
The connected domain is used to determine how many circle centers are there, that is, there are several light spots, and find the pixel coordinate position of the center of the white circle. By performing gradient processing on the image after thresholding, the resulting gradient release is shown in Figure 4.

\section{Figure 4 Gradient map}

Figure 5 shows the result of threshold processing and filling of the gradient map. Figure 5 (a) is an image of a light spot after processing the gradient map with a threshold of 50, and fills the gradient map after threshold processing, as shown in Figure 5 (b). After binarization, the black background pixel values will all become zero, and the pixel values at the white dots are all 1, and the area in formula (5) is directly calculated by counting the number of ones. Respectively count the number of pixels of the white circle in Figure 5(a) and Figure 5(b). Use statistical methods to calculate the areas of $S_{1}$ and $S_{2}$, and then use equation (5) to find the defocus radius R The values are 4.8055 pixels, 5.024 pixels and 4.8048 pixels, respectively.

\section{Figure 5 Thresholding and filling results of gradient map}

Four cameras with fixed viewing angles simultaneously collected three low-speed moving light-emitting balls, a total of four groups of images, each group of images contains 337 frames of out-of-focus blur images, using the algorithm proposed in this paper to calculate the spots in the out-of-focus blur images. The defocus blur radius is obtained, and the result of the defocus blur radius is shown in Figure 6.

\section{Figure 6 The blur radius distribution of the light spots in the four cameras}

Analyzing Figure 6 it is not difficult to find that the three facula images taken by four cameras all have a blur radius of 0 , which means the estimation fails. This is directly related to the fact that the facula size is too small and the imaging quality is low, which leads to serious information loss. Especially the out-of-focus blur image taken by the second camera, this phenomenon is more serious. Figure 7 shows a schematic diagram of the failure of the defocus blur radius estimation. It can be seen that the original circular spot imaging is approximately a rectangular spot. In order to better process the out-of-focus blurred image in the follow-up, the sample shown in Figure 7 should be avoided as much as possible during the data acquisition stage.

\section{Figure 7 Schematic diagram of failure to estimate the defocus blur radius}

\section{Results and discussion}

\section{1 Three-dimensional reconstruction based on double hidden layer BP neural network}

In recent years, related researches on artificial neural networks have continued to deepen, and have been applied in artificial intelligence, industrial manufacturing, management control, smart cities, medical diagnosis, economic decision-making and other fields, successfully solving many complex problems.

This paper uses the double hidden layer BP neural network to autonomously learn the relationship between the out-of-focus blurred image and the target position, and proposes a new end-to-end target position estimation algorithm. The double hidden layer BP network is a classic artificial neural network. When the network is trained, the signal is forwarded and the error is back-propagated, which has a good effect in practical applications. Figure 8 shows the two-hidden-layer BP neural network proposed in this paper for the four-view image. The input layer of the neural network has $12(4 \times 3)$ neurons, and the number 4 represents the number of cameras. The number 3 represents the pixel coordinate $\left(u_{i}, v_{i}\right)$ and the corresponding defocus radius $R_{i}$ of the center of each spot. The form of the pixel coordinate and the defocus radius is convenient for the subsequent double hidden layer BP neural network to read the data. There are 2 hidden layers, containing 20 neurons and 10 neurons respectively. The output layer is composed of 3 neurons. The number 3 represents the spatial coordinate $\left(x_{i}, y_{i}, z_{i}\right)$ of each spot. 


\section{2 Experimental result}

In the experiment, the training data set contains $4 \times 250$ images, and the test data set consists of $4 \times 20$ images. Each image contains 3 light spots. The training uses the LM algorithm. The specific error curve during the training process is shown in Figure 9 11 shown. Figure 9 shows the training state diagram of the double hidden layer BP neural network, Figure 10 shows the training performance diagram of the double hidden layer BP neural network, and Figure 11 shows the regression analysis diagram of the double hidden layer BP neural network. It can be seen from the figure that the algorithm converges quickly, and the algorithm gradually converges after many trainings.

Figure 9 Training state diagram of double hidden layer BP neural network

\section{Figure 10 Training performance graph of double hidden layer BP neural network}

\section{Figure 11 Double hidden layer BP neural network regression analysis diagram}

Use the trained double hidden layer BP neural network to estimate the spatial position of the input image. For 20 sets of test images, the proposed defocus blur parameter estimation method is used to estimate the defocus blur radius, and input the trained network model to predict the space coordinate value corresponding to the light spot. In order to verify the effect of the algorithm proposed in this paper on the 3D reconstruction of defocused blurred images, the defocused blur radius value is fixed to 0 during the training of the double hidden layer BP neural network, that is, the reconstruction obtained without considering the defocused blur radius (no blur radius) The result is compared with the reconstructed result when the defocus blur radius (with blur radius) is considered. Figure 12 is a comparison diagram of the results of the three-dimensional reconstruction of the real position with and without the blur radius. It can be seen from the figure that most of the predicted position and the real position can be better overlapped, some deviations between the predicted position and the real position, and only a few have large deviations. Comparing the three-dimensional reconstruction results with defocus blur radius and no defocus blur radius, it can be seen that the reconstruction accuracy is higher when the defocus blur radius is considered, which shows that the double hidden BP neural network constructed in this paper can learn The relationship between the out-of-focus blurred image and the spatial position has certain practical value.

\section{Figure 12 Comparison of predicted coordinates and real coordinates with/without fuzzy radius}

In the following, the method in [14] is used as the root mean square error between the real coordinates and the predicted coordinates to quantitatively illustrate the accuracy of the three-dimensional coordinate estimation algorithm of the defocused target based on the double hidden layer BP network model in this paper. Figure 13 shows the change trend of the root mean square error obtained by comparing the root mean square error of the 3 light spots with the defocus blur radius and no defocus blur radius with the real position in the 20 test defocus blur images. It can be seen that the defocus blur radius is considered The variation of the root mean square error of the three spots is smaller than the root mean square error of the three spots when the defocus blur radius is not considered. This shows that under the condition of the same external imaging environment, when the three-dimensional reconstruction of the defocused blur image spot is performed, Considering the defocus blur, the accuracy of 3D reconstruction is improved compared with not considering the defocus blur. Calculate the average value of the root mean square error of the reconstruction of the 3 light spots with and without the blur radius. The results are shown in Table 1. It can be seen that the average error of the 3D reconstruction of the 3 light spots is all when considering the defocus blur radius. It becomes smaller, which once again shows that the algorithm in this paper has a better effect on the $3 \mathrm{D}$ reconstruction of defocus blurred images.

\section{Figure 13 The actual position and the change of position error with (without) fuzzy radius}

Table 1 Comparison of the average error of the three light spots without blur radius and with blur radius

\begin{tabular}{|l|l|l|}
\hline & No blur radius/pixel & with blur radius/pixel \\
\hline No. 1 spot & 21.1195 & 10.9191 \\
\hline No. 2 spot & 16.0178 & 9.5859 \\
\hline
\end{tabular}




\begin{tabular}{|l|l|l|}
\hline No. 3 spot & 20.4728 & 12.2045 \\
\hline
\end{tabular}

\section{Conclusion}

In this paper, the three-dimensional reconstruction of the target spot under the defocus blur is carried out. First, the disc defocus blur model is given and the defocus blur radius is estimated. 然 After that, a double hidden layer BP neural network is used to take the center pixel coordinates of the defocused spot and the defocus radius of the spot as the input of the network, and the three-dimensional coordinates of the spot as the output for training. Finally, the reconstruction result considering the defocus radius is compared with the result of direct reconstruction without considering the defocus radius. The experimental results show that the algorithm proposed in this paper improves the reconstruction accuracy of defocused blur spots.

\section{Availability of data and material}

Data sharing not applicable to this article as no datasets were generated or analysed during the current study.

\section{Competing interests}

The authors declare that they have no competing interests in this section.

\section{Funding}

This work was supported by the National Natural Science Foundation of China under grant no. 51575276, no52075258.

\section{Authors' contributions}

Shi Yun wrote the program and the manuscript of the thesis, also participated in the research design and conducted statistical analysis. Professor Zhu

Yan-yan conceived this research, participated in its design and coordination, and helped revise the man uscript. The final manuscript read and approved by all authors.

\section{Abbreviation}

1.3D:3 Dimensions

2.BP:back propagation

3.Coc:Circle of Confusion

\section{references:}

[1] Zheng Chujun, Li Rong, Chang Hongsen. Restoration of defocus blurred digital image using wiener filter in frequency domain [J]. Laser Journal, 2004, 25(5):57-58.

[2] Zhou Luoyu, Zhang Bao, Yang Yang. Estimation of parameter of defocused blurred image using Hough transform [J]. Infrared and Laser Engineering, 2012, 41(10):2833-2837.

[3] JIANG Ming-Yong, CHEN Xiang-ning, YU Xia-qiong. A blind restoration method for defocus blurred remote sensing imagery [J]. Science of Surveying and Mapping, 2012, 37(4):135-137.

[4] Liang Min, Zhu Hong. Defocus blur parameter estimation method based on blur spectrum characteristic of image edge [J]. Journal of Computer Applications, 2014, 34(4):1177-1181.

[5] Jiang Y G, Wu Q, Guo P. Defocused image restoration using RBF network and Kalman filter[C]// Proceedings of the 2005 IEEE international conference on systems, man and cybernetics, 2005:2507-2511.

[6] Su L Y, Li F L, Xu F, et al. Defocused image restoration using RBF network and iterative Wiener filter in wavelet domain[C]// Proceedings of the first international congress on image and signal processing. 2008:311-315.

[7] Moghaddam M E. A robust noise independent method to estimate out of focus blur [C]// Proceedings of the IEEE international conference on acoustics, speech, and signal processing. 2008:1273-1276.

[8] REDERTA, HENDRIKS E, BIEMOND J. Correspondence estimation in image Paris [J]. IEEE signal processing magazine, 1999, 16(3):29-46.

[9] Xu Bin, Yu Mei, Ye Xien, etc. A fast disparity estimation algorithm for stereo video coding based on H.264. [J]. Journal of Zhejiang University (Science Edition), 2006, 33(4):412-416.

[10] KIM Y, KIM J, SOHN K. Fast disparity and motion estimation for multi-view video coding [J]. IEEE transactions on consumer electronics, 2007, 53(2):712-719.

[11] Fan Haiju, Feng Yunzhi, Wang Tao, Feng Naiqin. New method of blurred radius detection in defocused image [J]. Journal of Computer Applications, 2012, 32(7):1875-1878. 
[12] Zhang Yang. Research on the Restoration and Processing Technology of Spatial Defocused Blurred Image [D]. University of Chinese Academy of Sciences, 2016.

[13] Yang Yawei, Geng Zhi, Wang Rui, et al. Spatially-Varying Out-of-Focus Deblurring of Conventional Camera [J]. Electronics Optics \& Control, 2015, 22(9):91-95.

[14] Bai Lizhi. Large field of view multi-target dynamic vision measurement technology [D]. Nanjing University of Aeronautics and Astronautics, 2021.

Figure legends

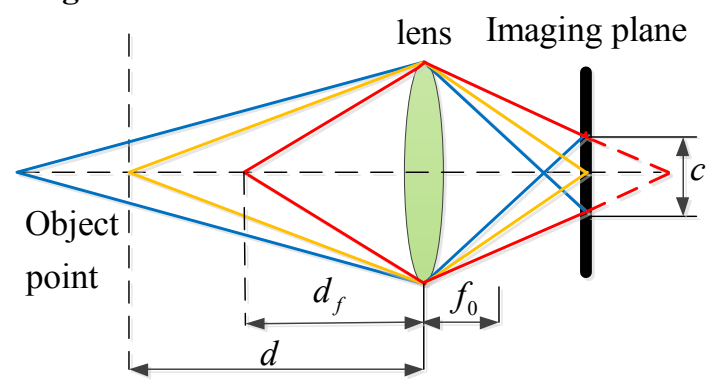

(a) Defocus blur formation principle

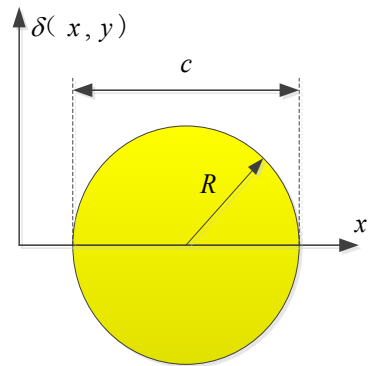

(b) CoC diagram based on disc mode

Figure 1 The relationship between the principle of defocus blur formation and CoC

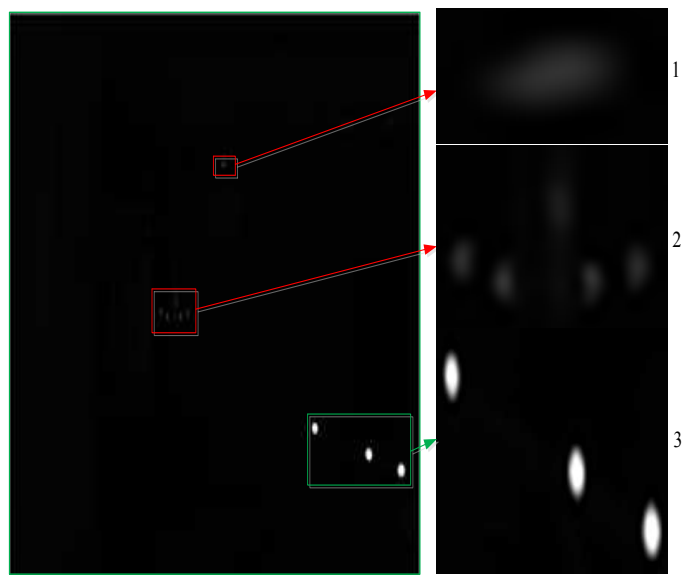

Figure 2 Actual image taken

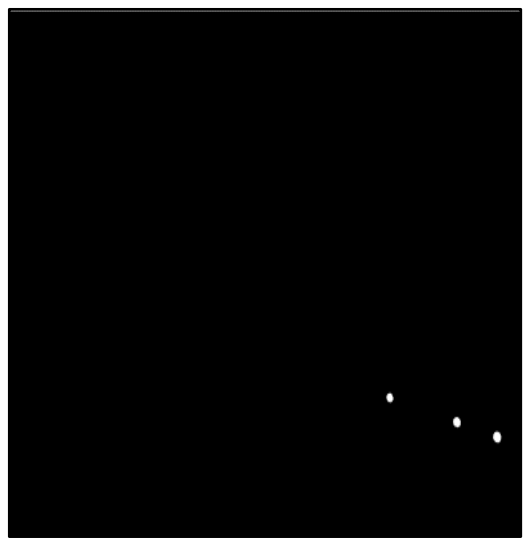

Figure 3 Threshold processing result graph 


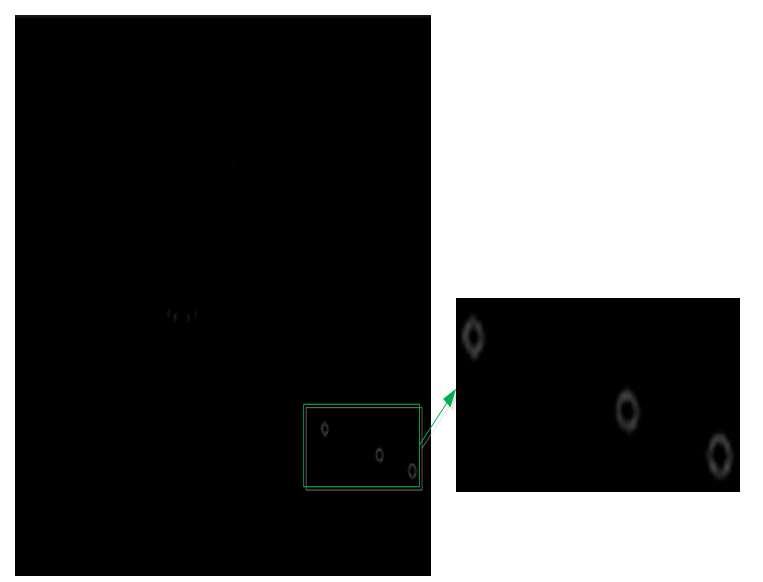

Figure 4 Gradient map

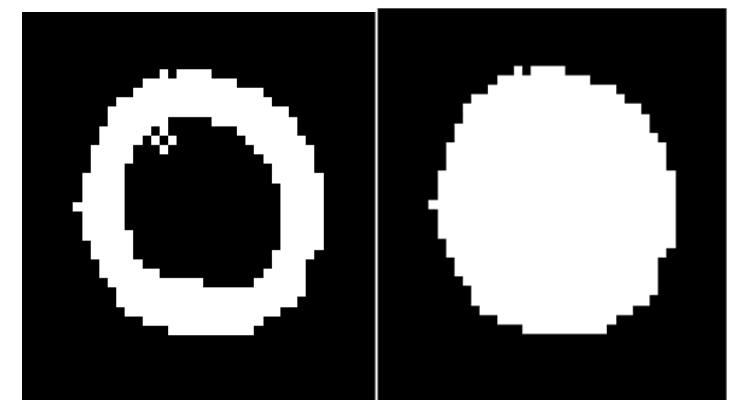

(a) Thresholding the gradient map (b) Fill map

Figure 5 Thresholding and filling results of gradient map 


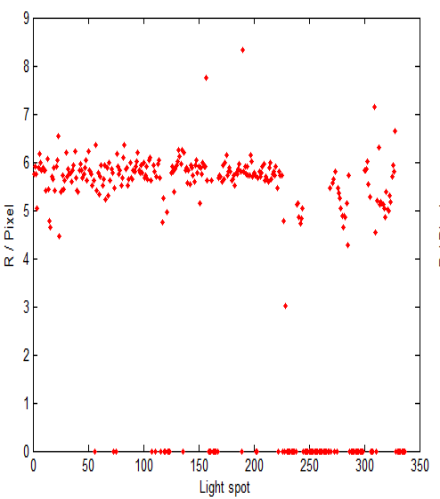

(a) Spot 1 in camera 1

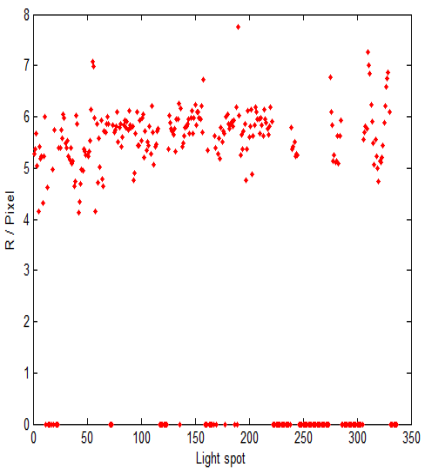

(d) Spot 1 in camera 2

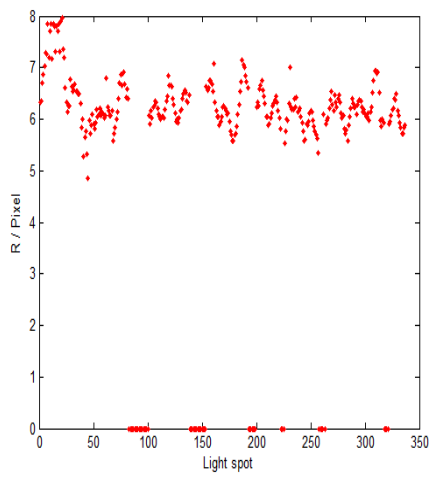

(h) Spot 1 in camera 3

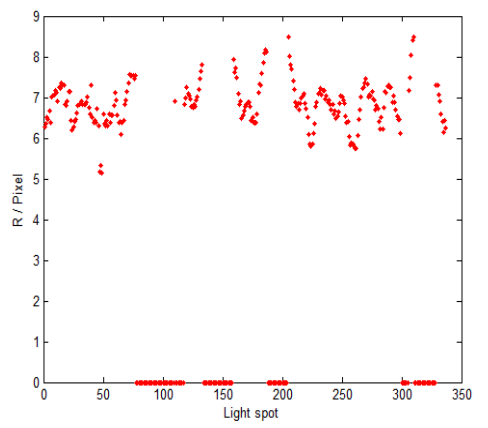

(k) Spot 1 in camera 4(1)

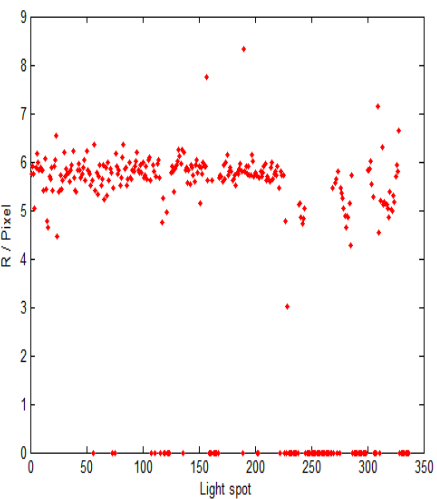

(b) Spot 2 in camera 1

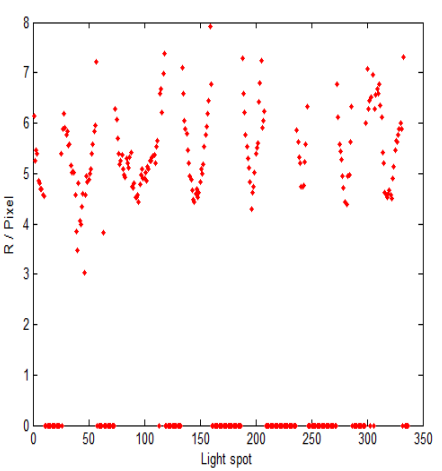

(e) Spot 2 in camera 2

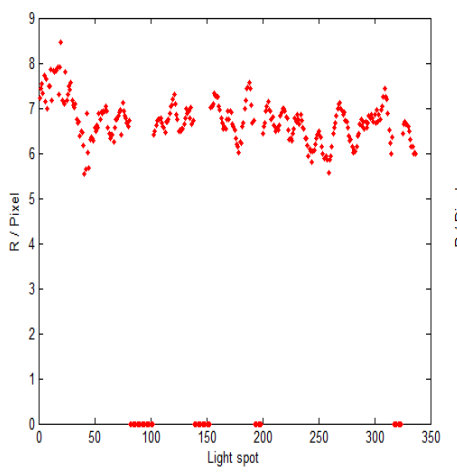

(i) Spot 2 in camera 3

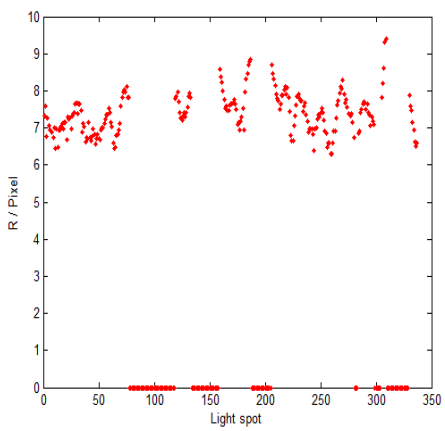

Spot 2 in camera $4(\mathrm{~m})$

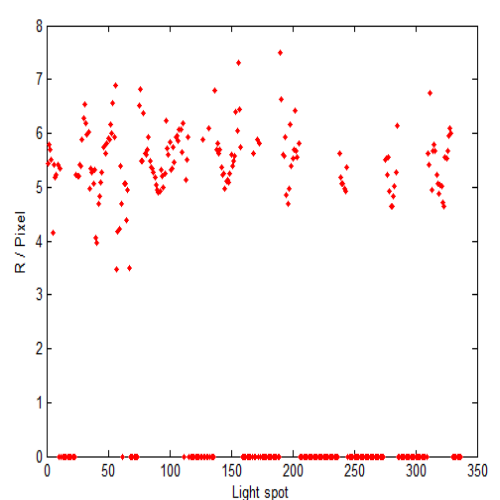

(c) Spot 3 in camera 1

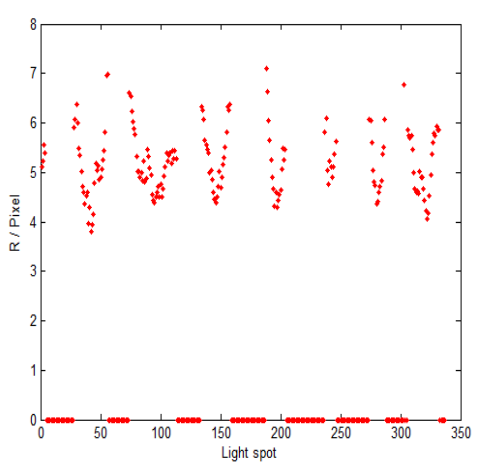

(f) Spot 3 in camera 2

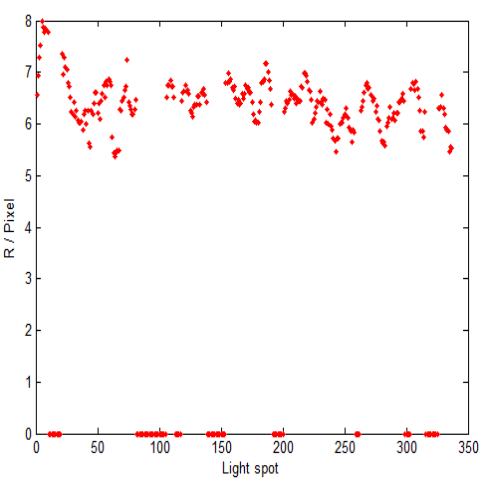

(j) Spot 3 in camera 3

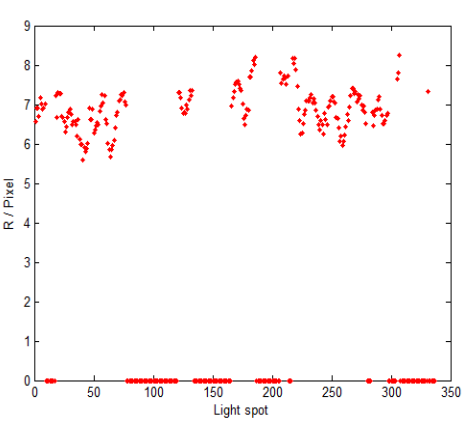

Spot 3 in camera 4

Figure 6 The blur radius distribution of the light spots in the four cameras 


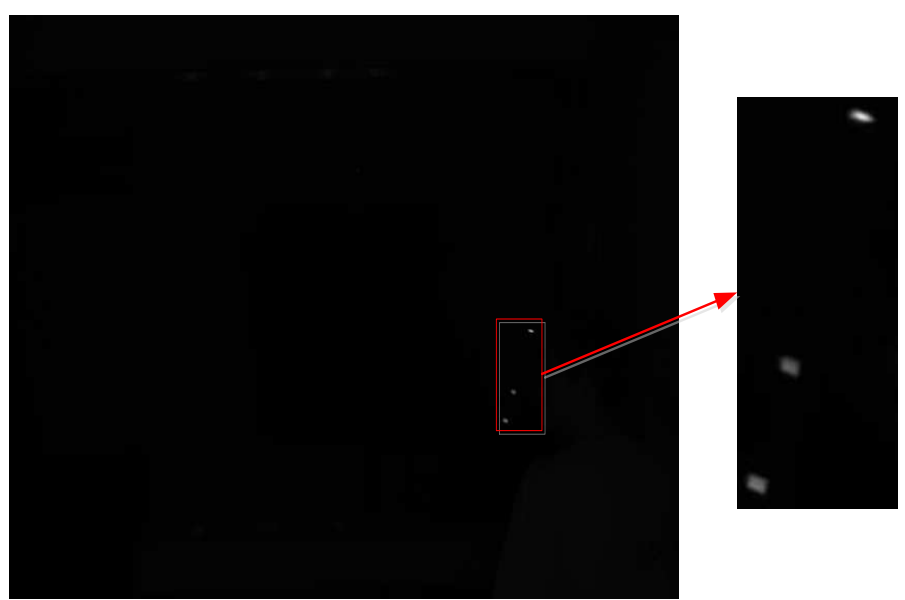

Figure 7 Schematic diagram of failure to estimate the defocus blur radius

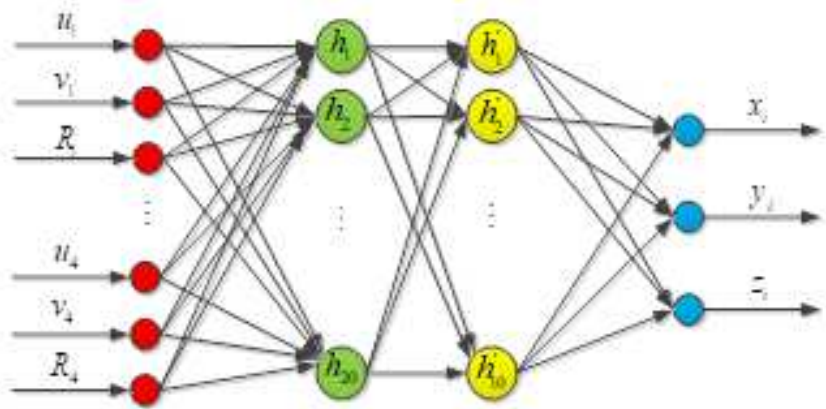

Figure 8 Defocused blur 3D reconstruction network structure diagram

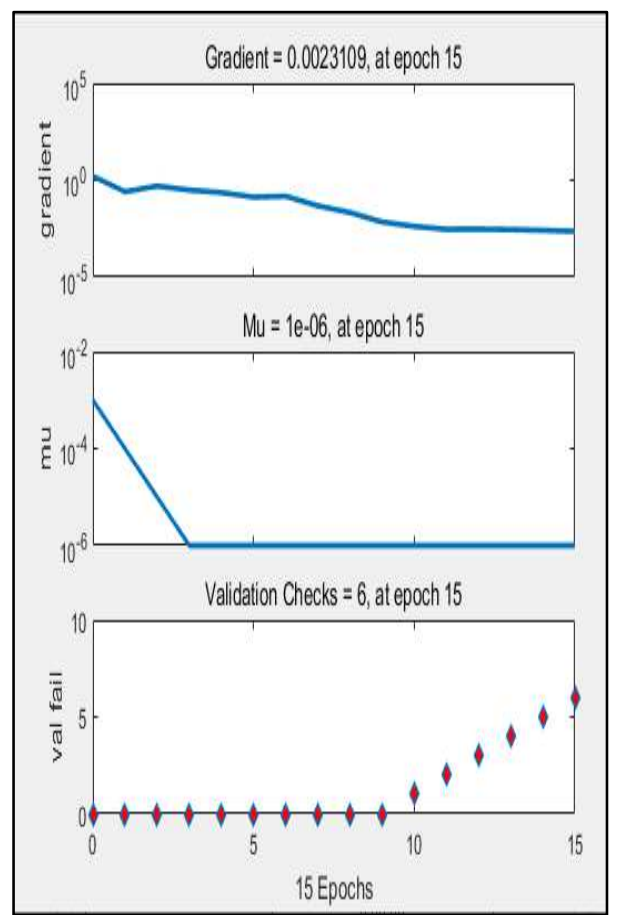

Figure 9 Training state diagram of double hidden layer BP neural network 


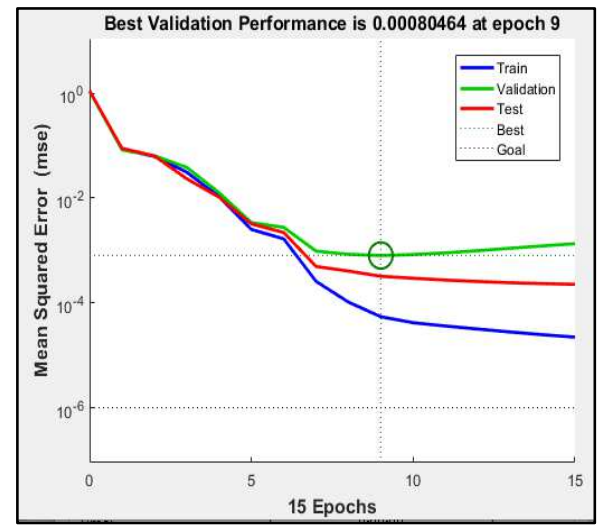

Figure 10 Training performance graph of double hidden layer BP neural network

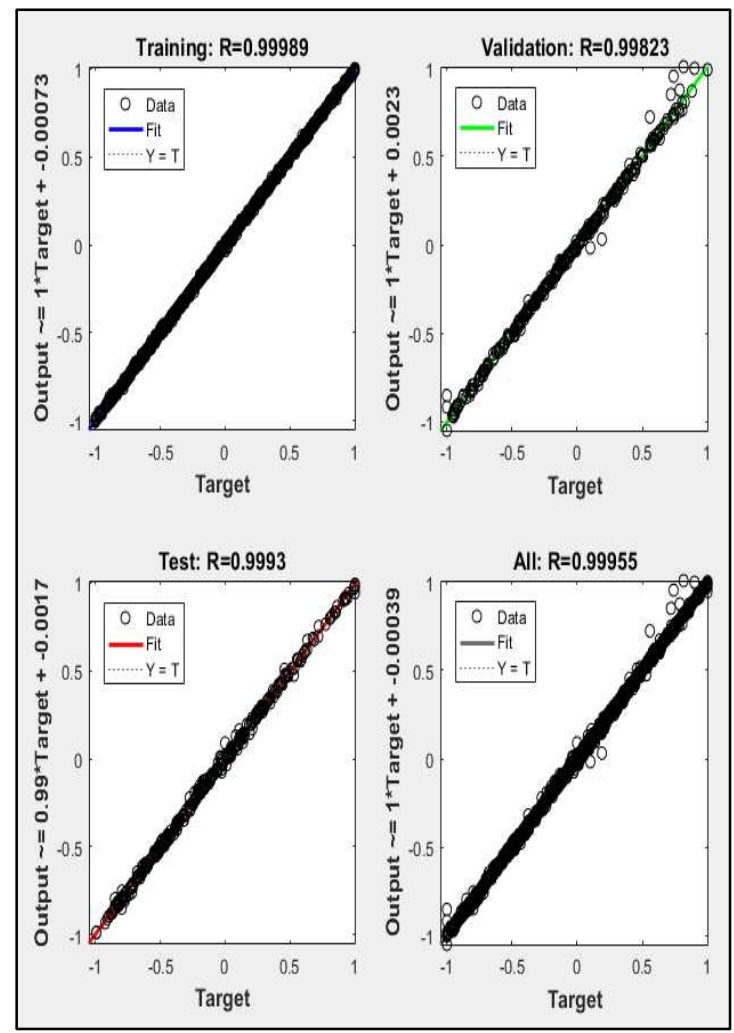

Figure 11 Double hidden layer BP neural network regression analysis diagram 

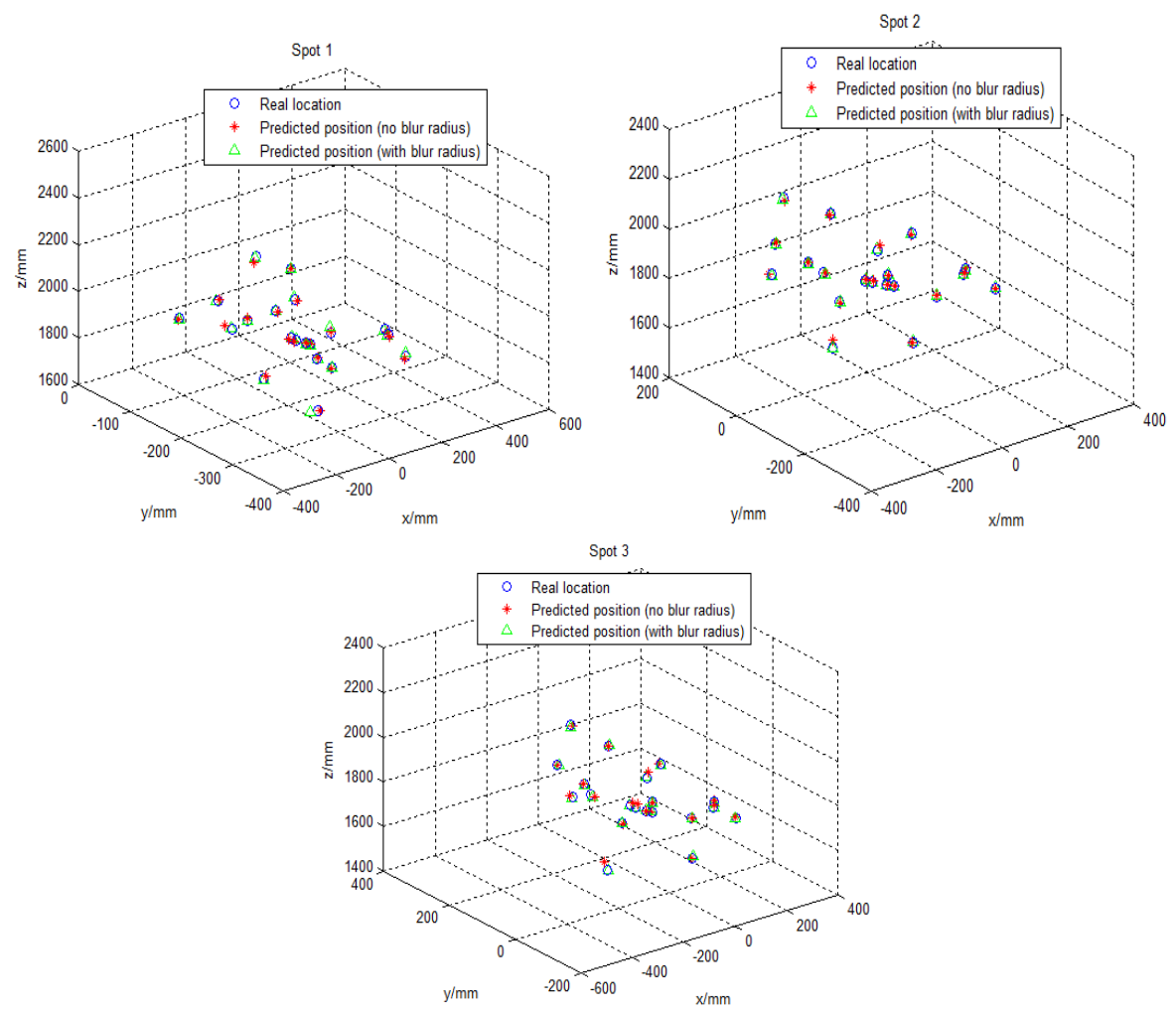

Figure 12 Comparison of predicted coordinates and real coordinates with/without fuzzy radius
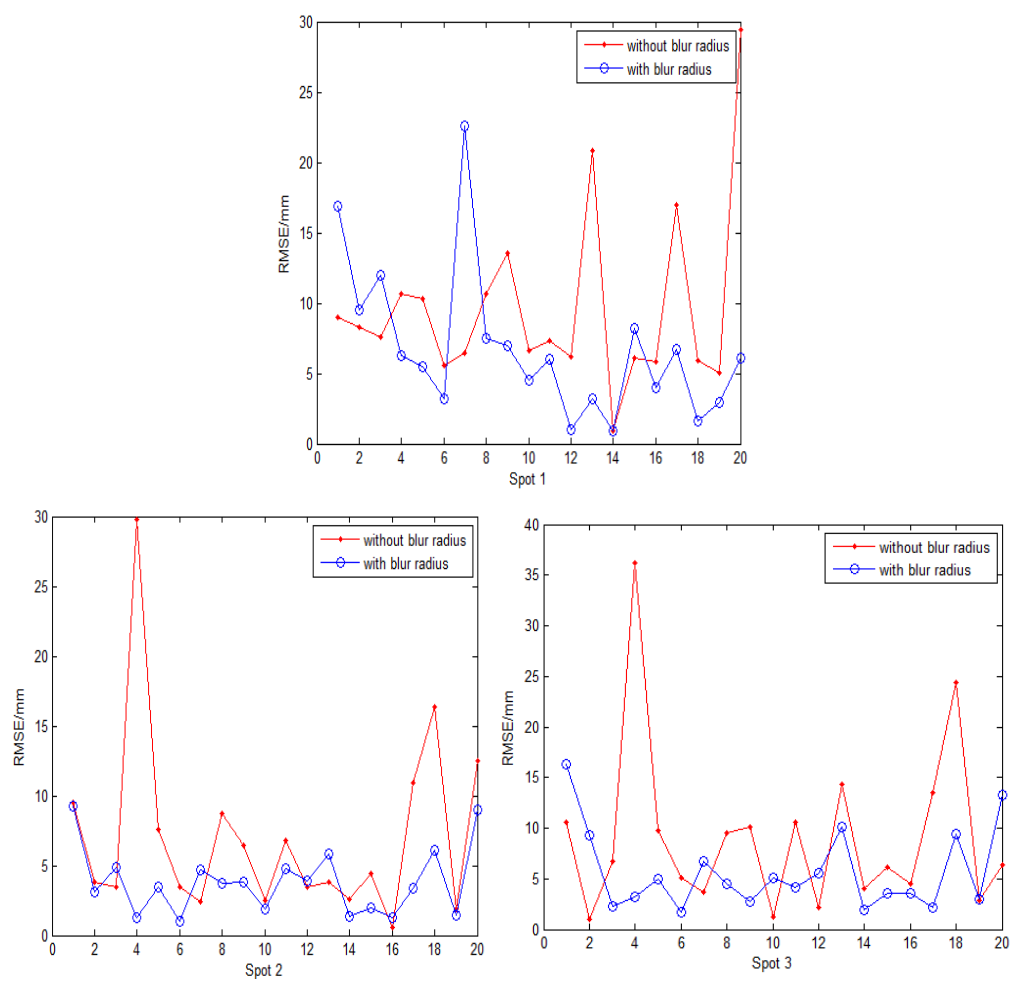

Figure 13 The actual position and the change of position error with (without) fuzzy radius 


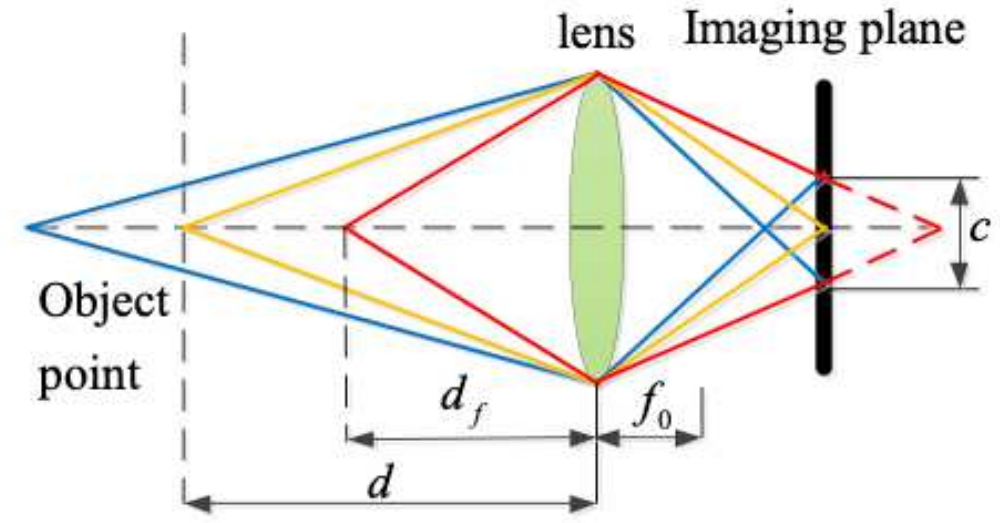

(a) Defocus blur formation principle

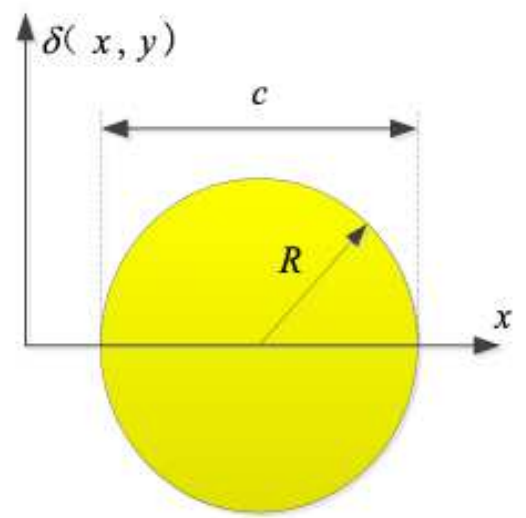

(b) $\mathrm{CoC}$ diagram based on disc mode

\section{Figure 1}

The relationship between the principle of defocus blur formation and CoC
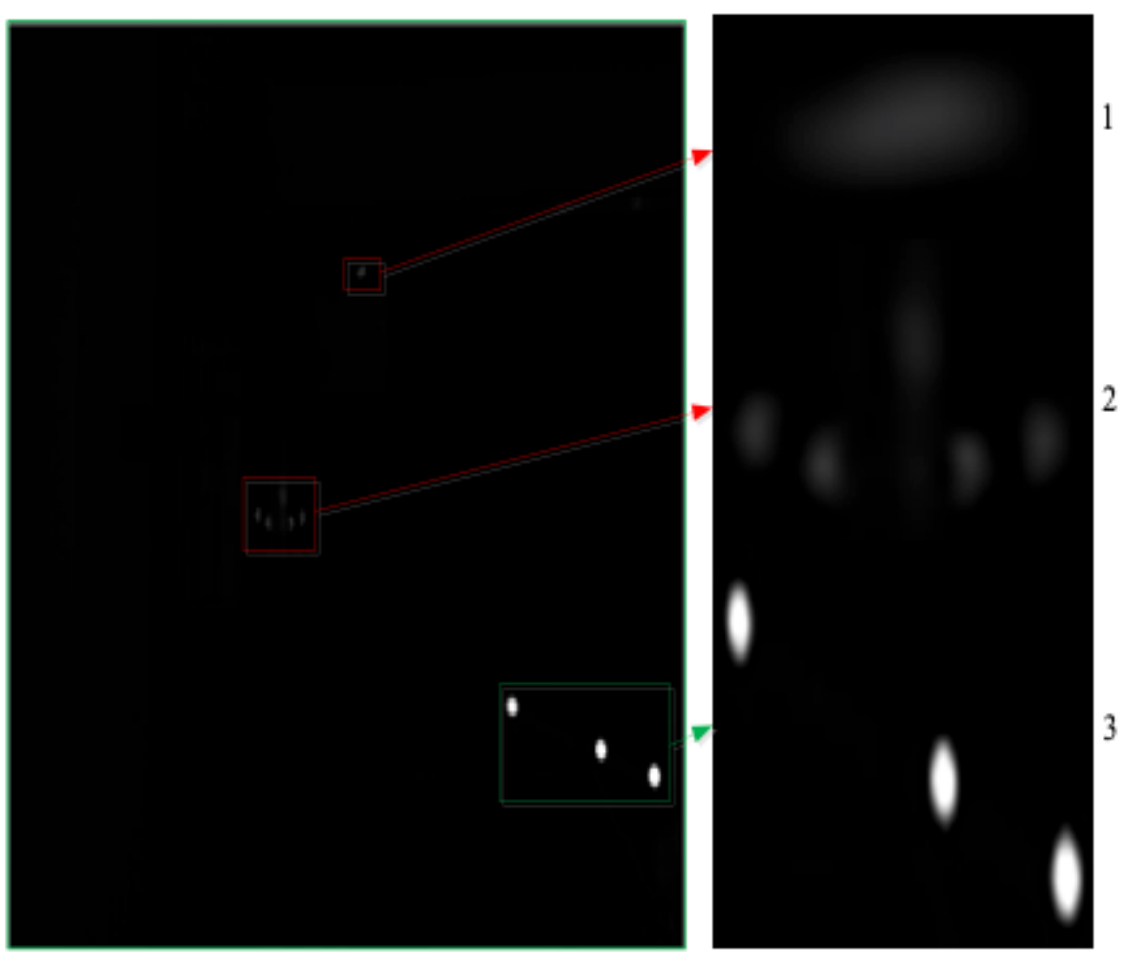

Figure 2

Actual image taken 


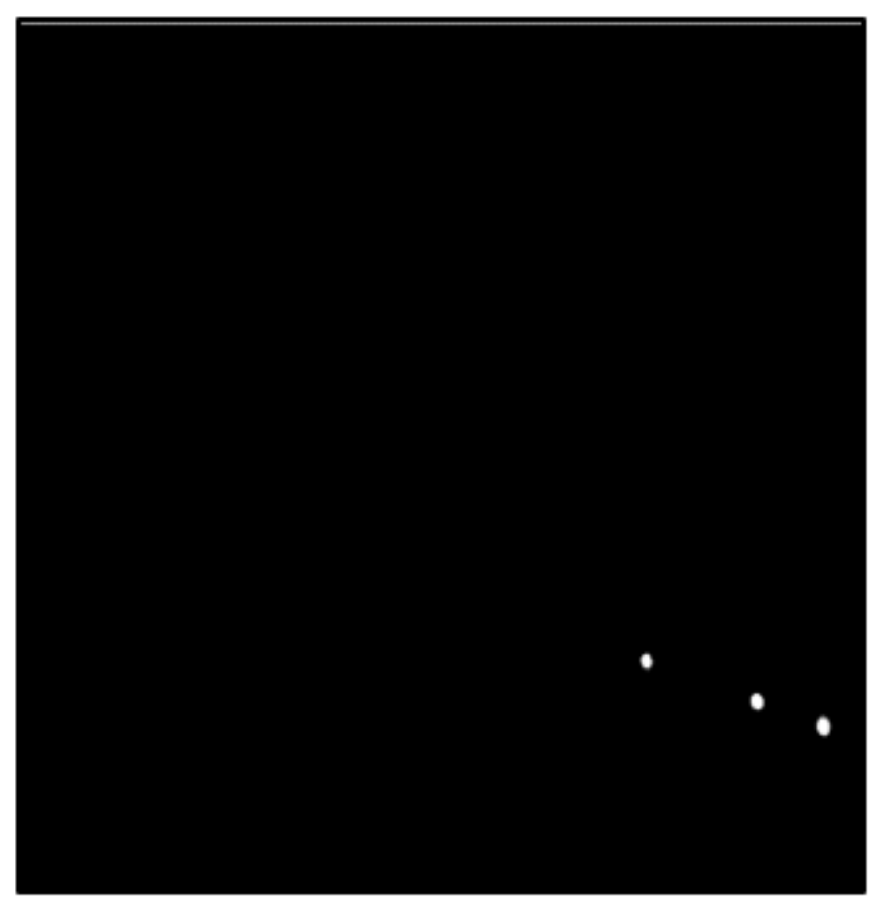

Figure 3

Threshold processing result graph

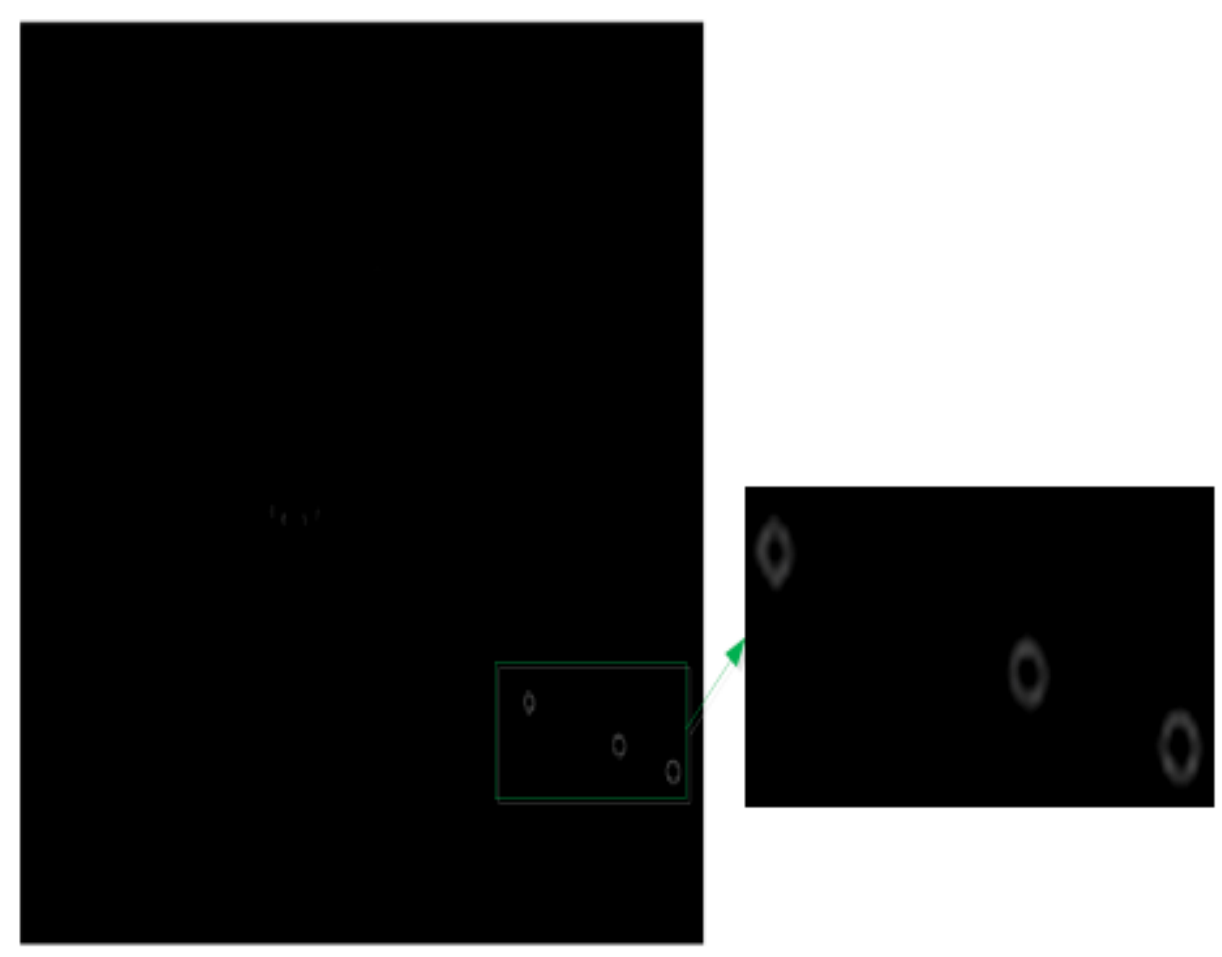

Figure 4

Gradient map 


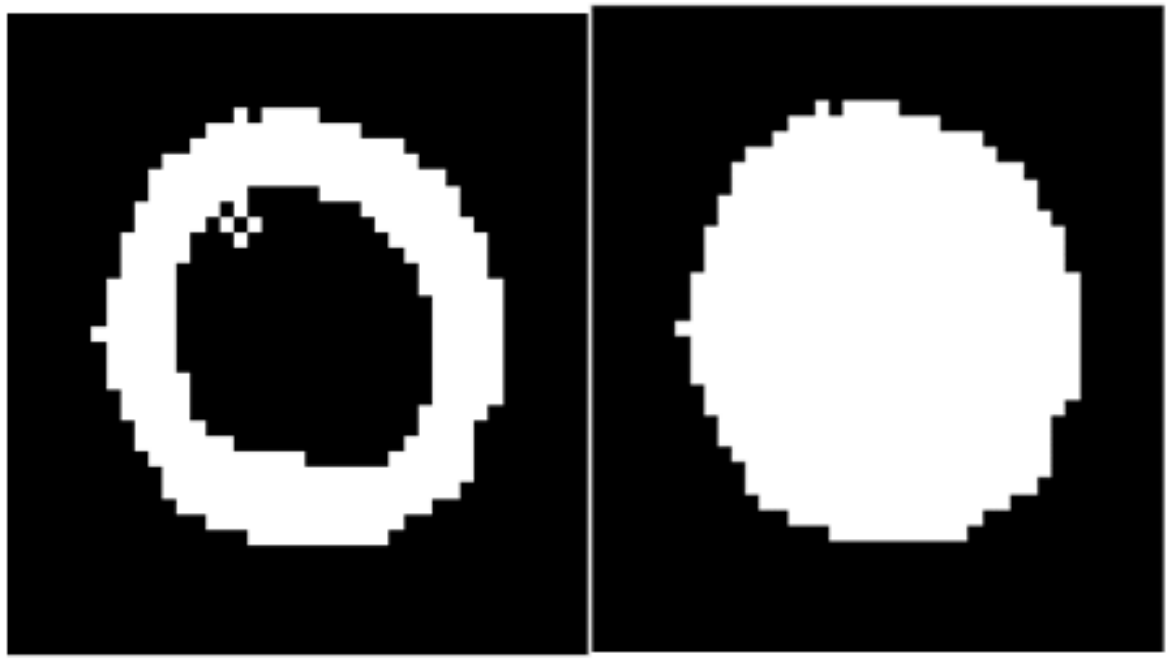

(a) Thresholding the gradient map

(b) Fill map

Figure 5

Thresholding and filling results of gradient map 


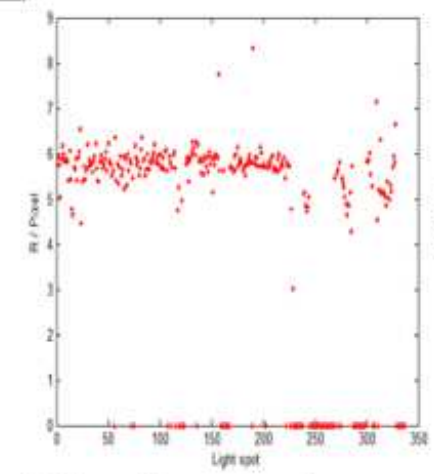

(a) Spot 1 in camera 1

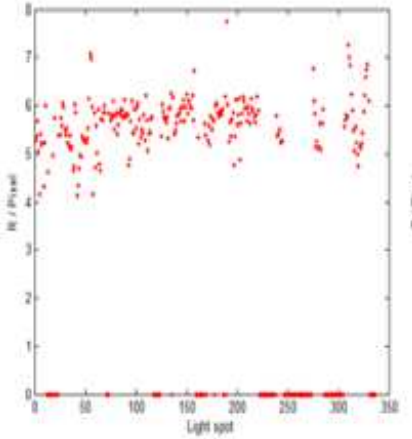

(d) Spot 1 in camera 2

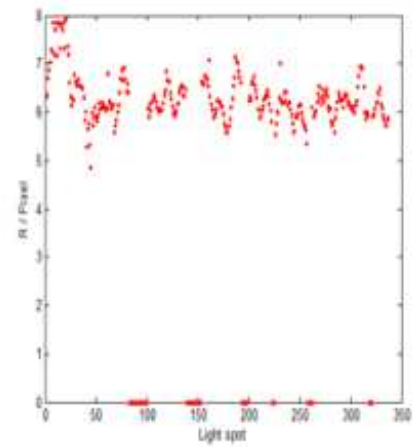

(h) Spot 1 in camera 3

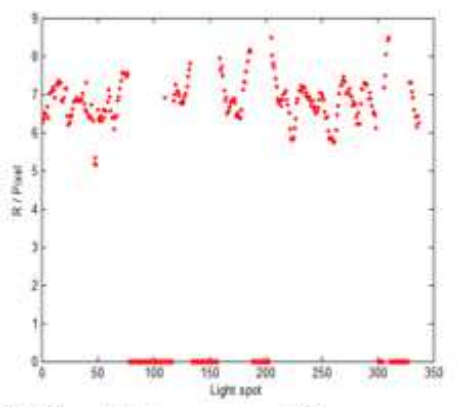

(k) Spot 1 in camera 4(1)

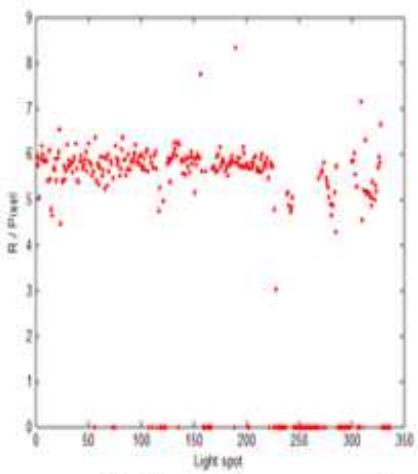

(b) Spot 2 in camera 1

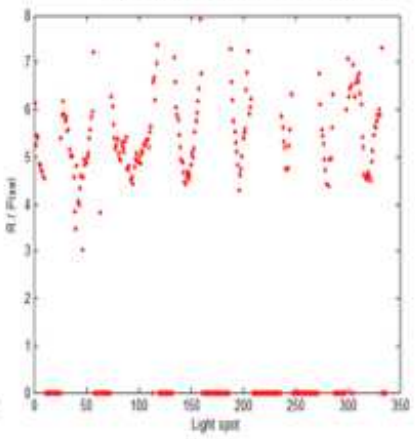

(e) Spot 2 in camera 2

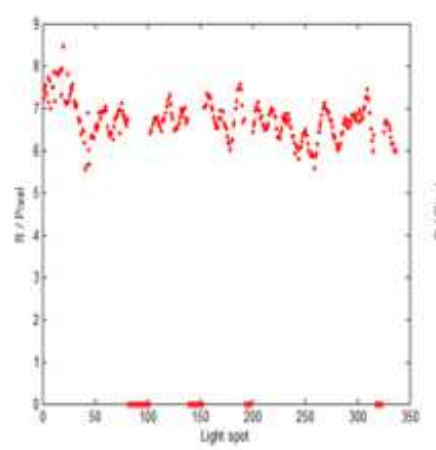

(i) Spot 2 in camera 3

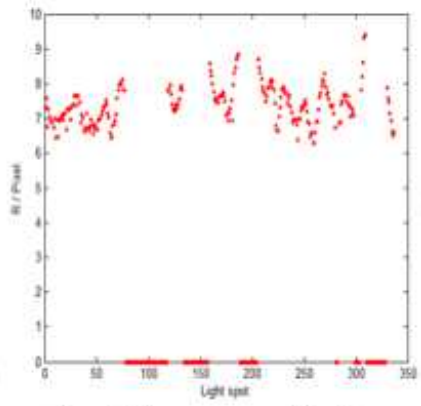

Spot 2 in camera $4(\mathrm{~m})$

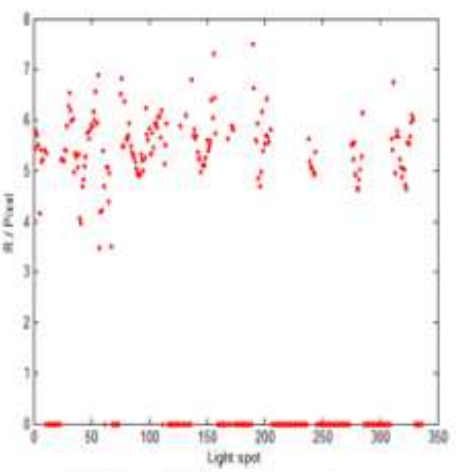

(c) Spot 3 in camera 1

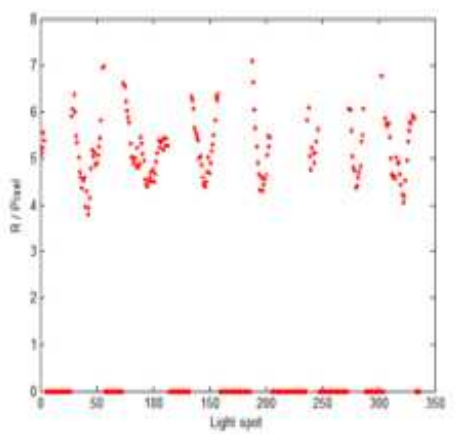

(f) Spot 3 in camera 2

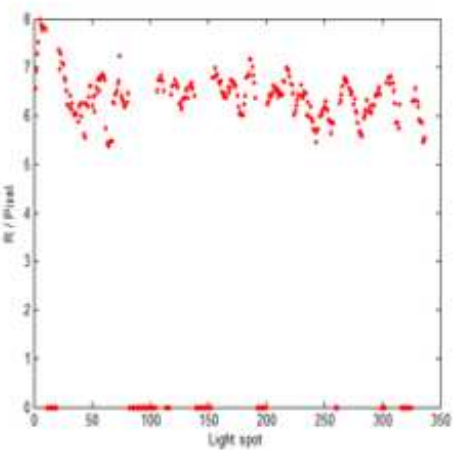

(j) Spot 3 in camera 3

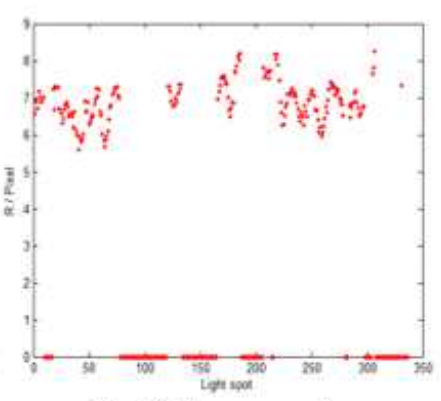

Spot 3 in camera 4

\section{Figure 6}

The blur radius distribution of the light spots in the four cameras 


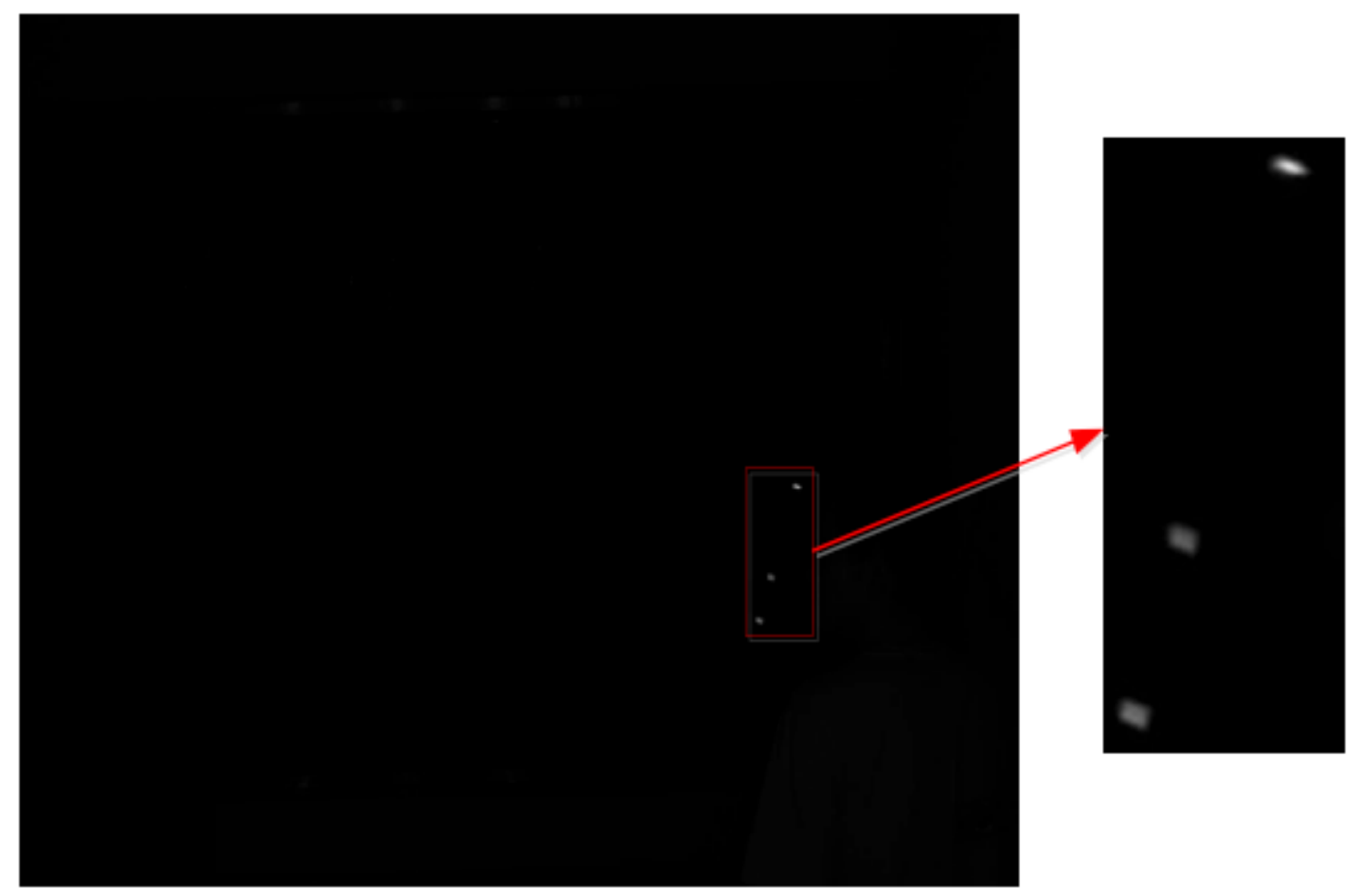

Figure 7

Schematic diagram of failure to estimate the defocus blur radius

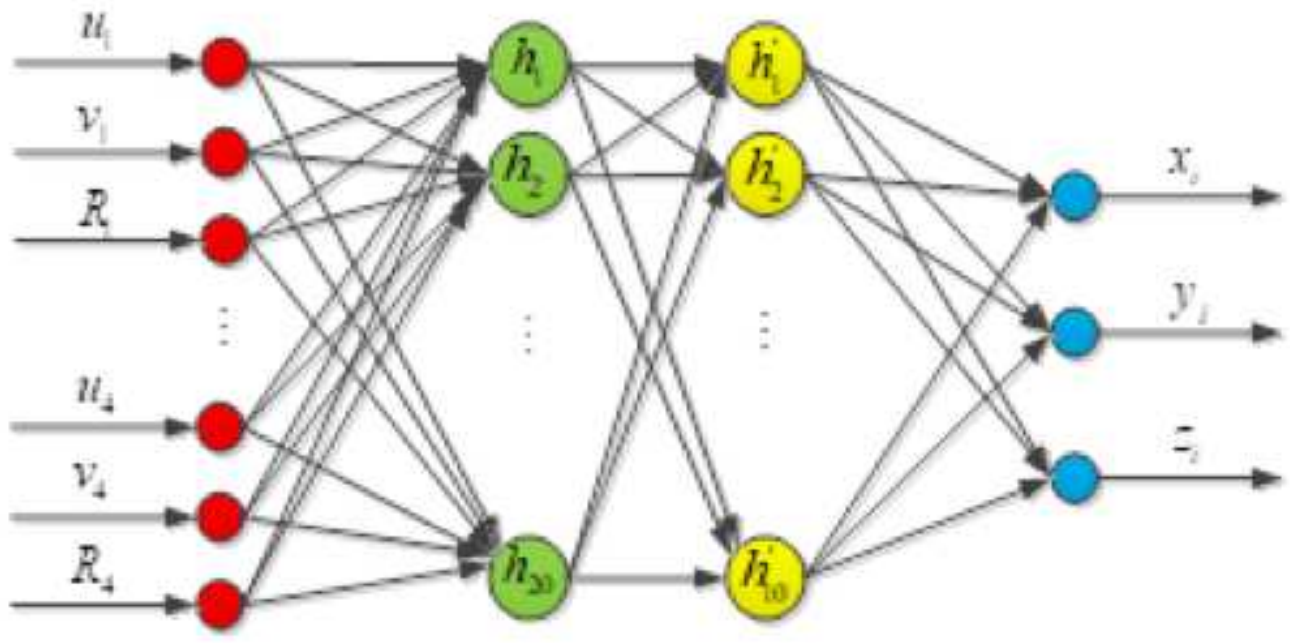

Figure 8

Defocused blur 3D reconstruction network structure diagram 


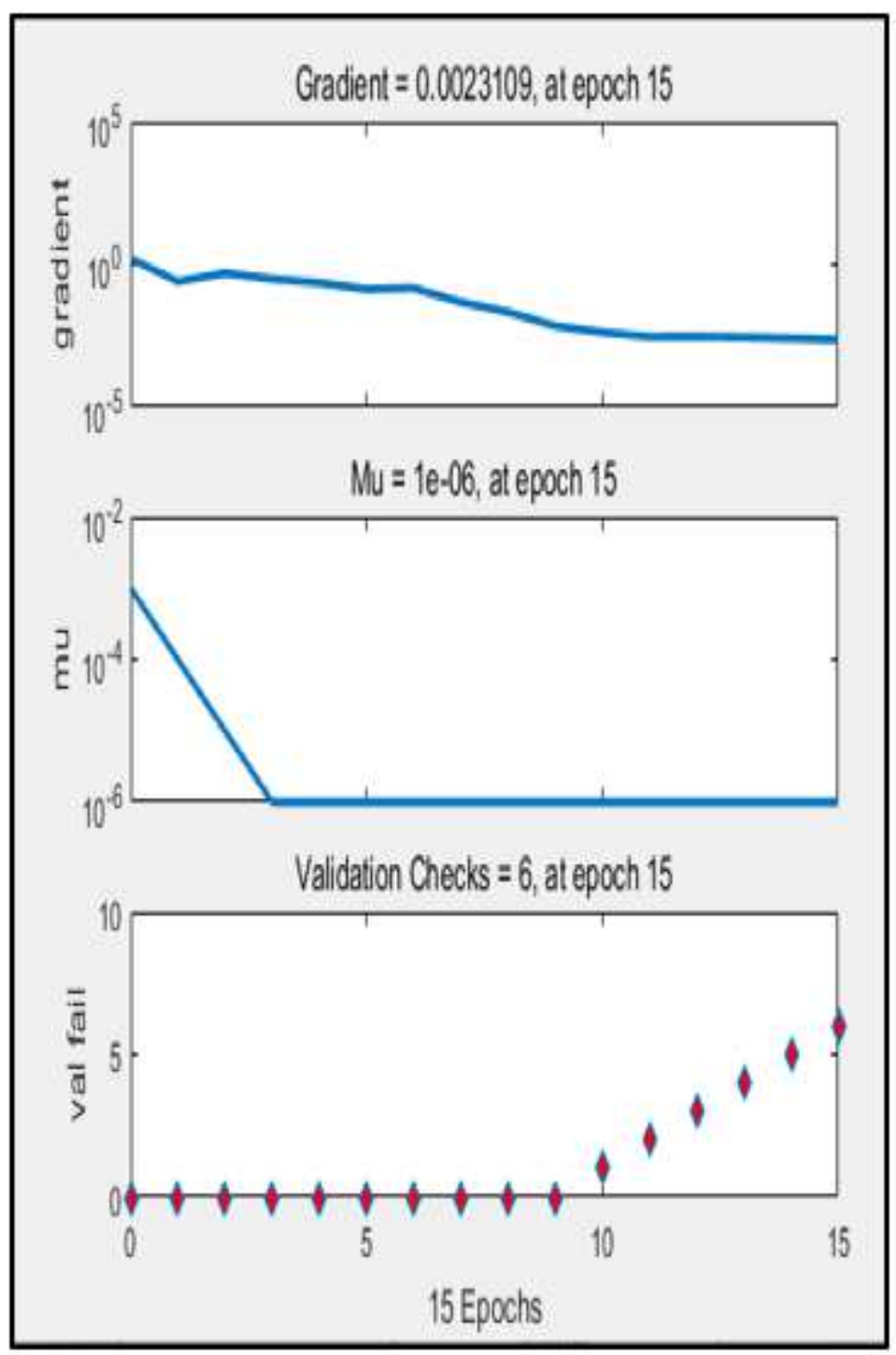

Figure 9

Training state diagram of double hidden layer BP neural network 


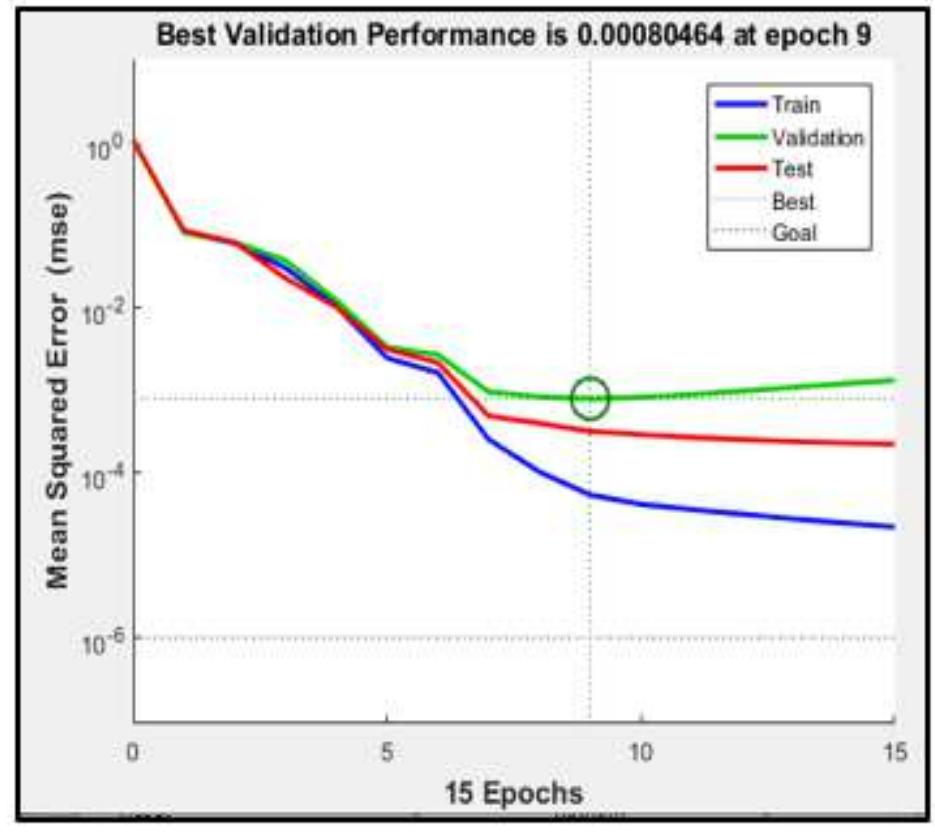

Figure 10

Training performance graph of double hidden layer BP neural network 


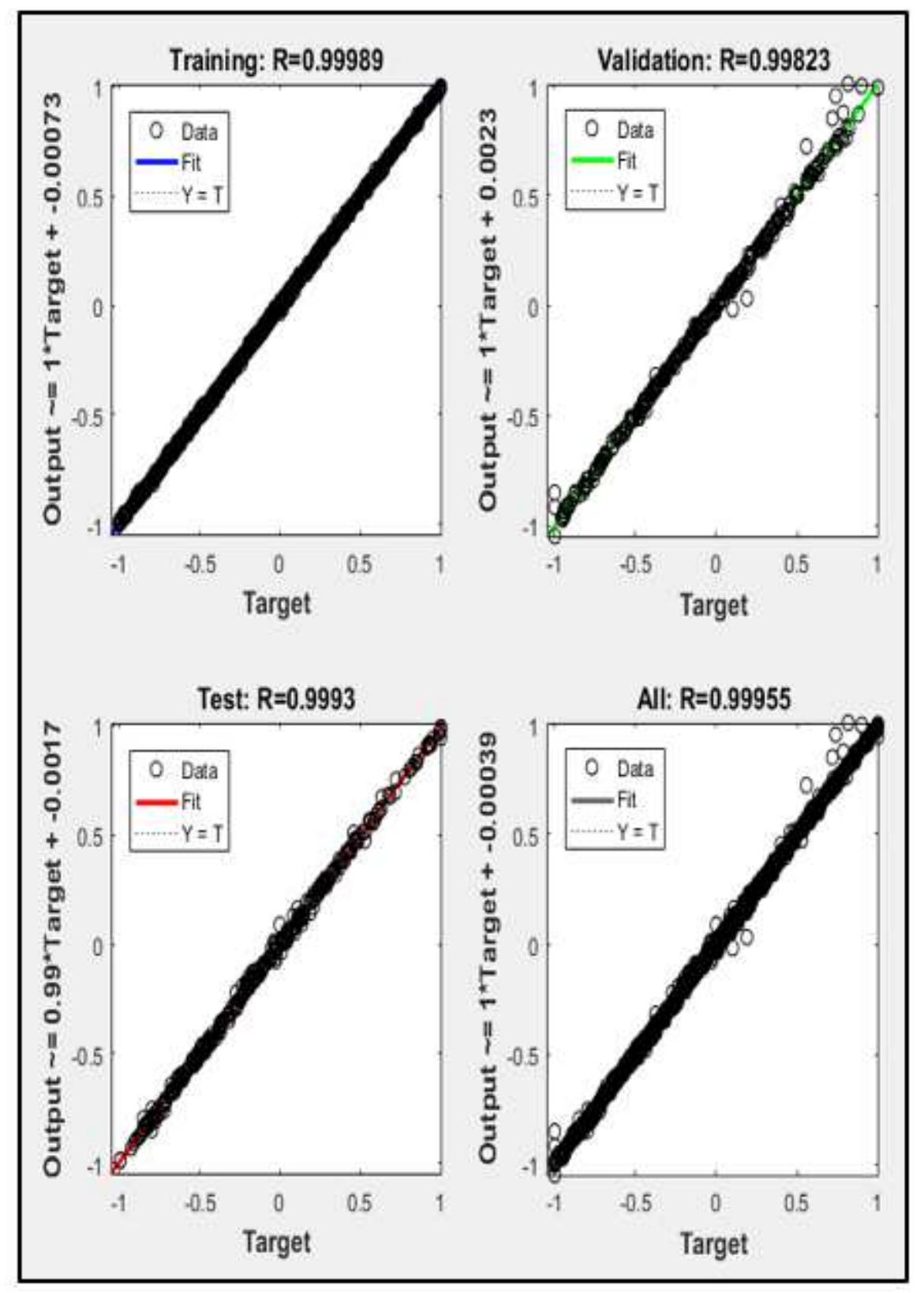

Figure 11

Double hidden layer BP neural network regression analysis diagram 

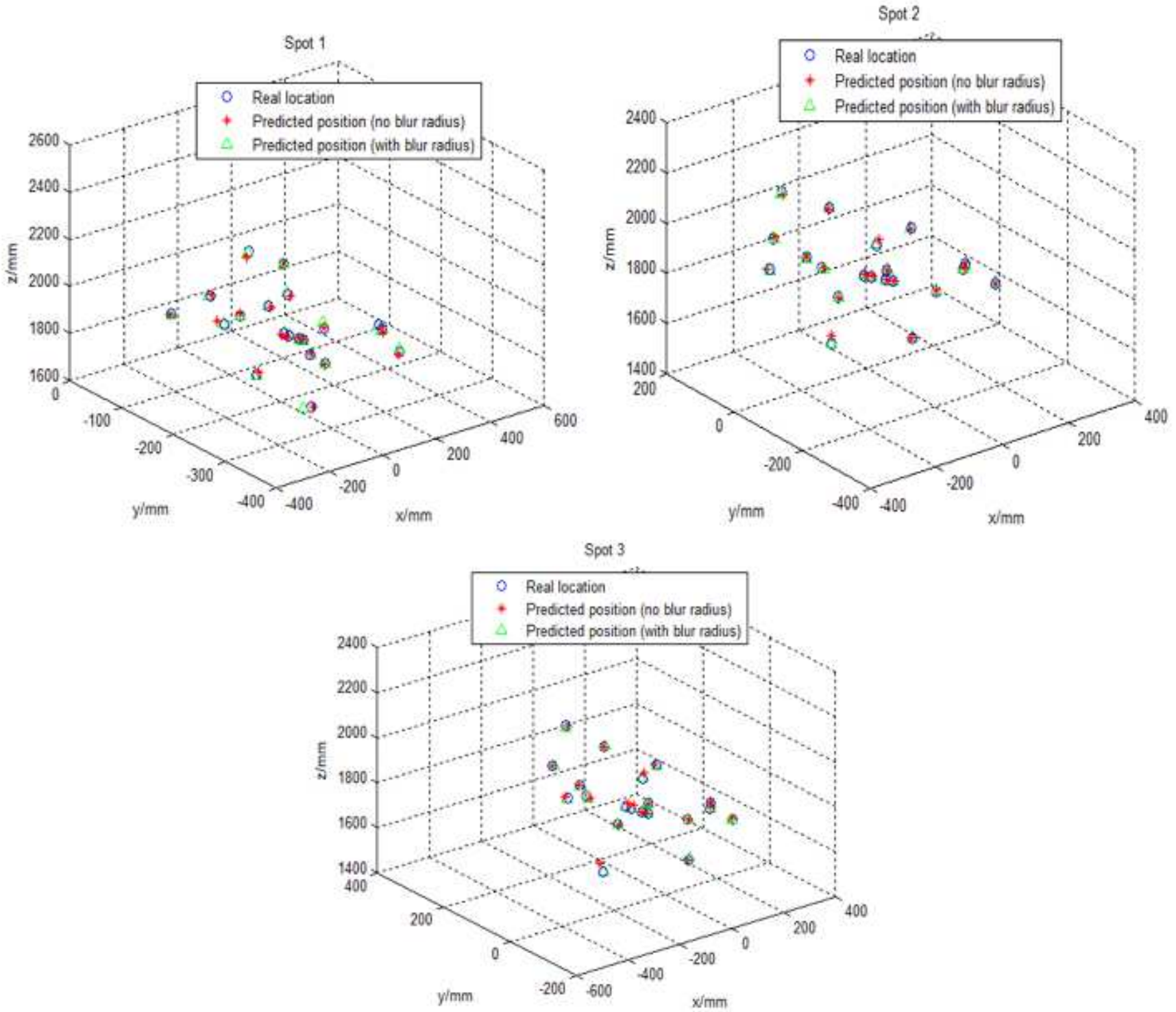

Figure 12

Comparison of predicted coordinates and real coordinates with/without fuzzy radius 

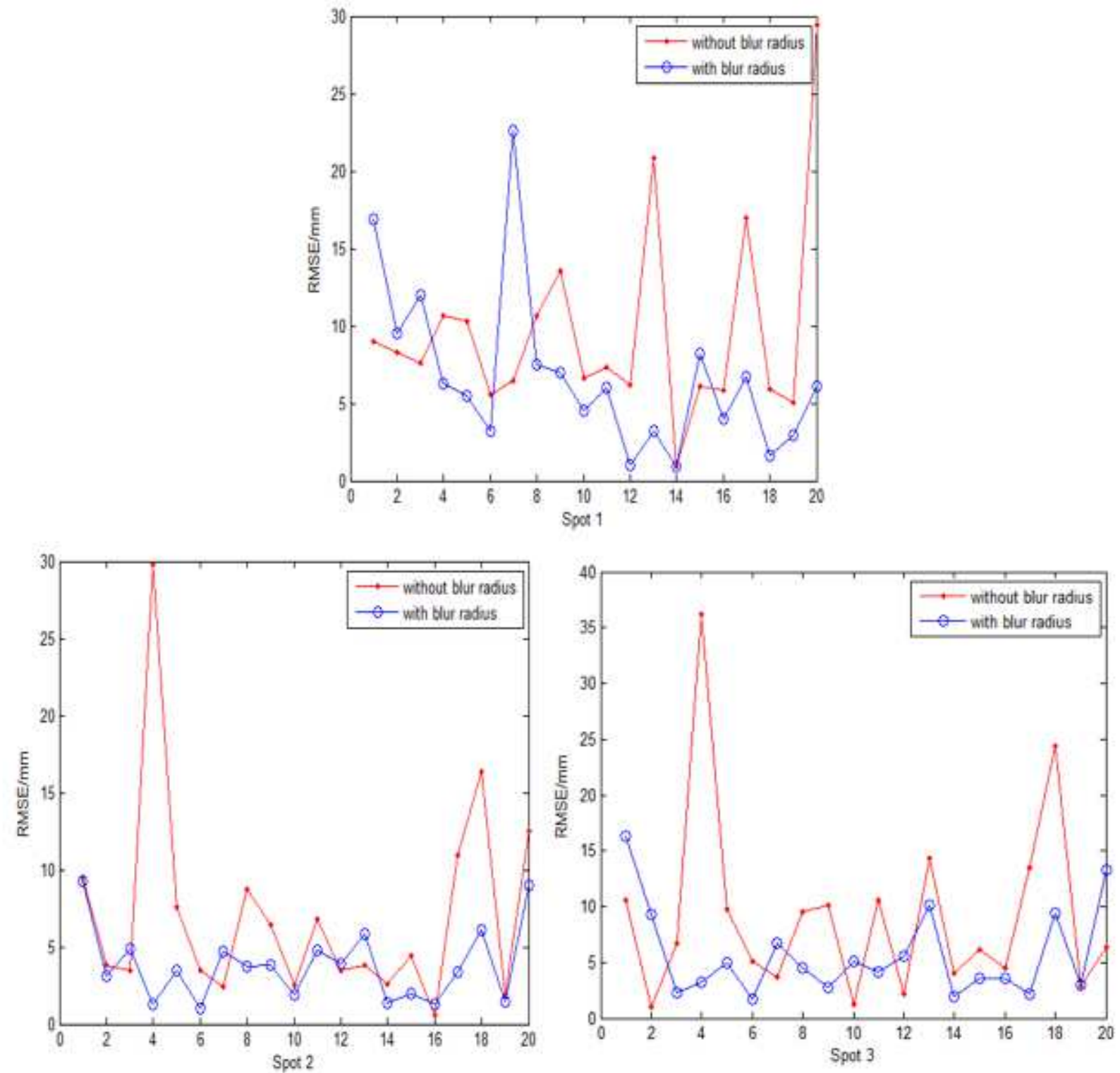

Figure 13

The actual position and the change of position error with (without) fuzzy radius 